\title{
Cortical Superficial Siderosis Presumed due to Cerebral Amyloid Angiopathy: Minimum Standards for Rating and Reporting
}

W ith great interest, I read the recent article by Inoue et $\mathrm{al}^{1}$ on the diagnostic significance of cortical superficial siderosis (cSS) for Alzheimer disease in a memory clinic setting. In line with other recent studies on the topic, the authors found cSS in 12 of 347 (3.5\%) patients in the memory clinic, a prevalence much lower compared with pathologically proved cerebral amyloid angiopathy (CAA) (approximately 50\%), but still higher than the $1 \%$ prevalence seen in population-based studies. Not surprisingly, in the current study, cSS was found to be associated with strictly lobar microbleeds, ${ }^{1}$ a putative marker of CAA.

In a letter to the editor about this study, Bai et $\mathrm{al}^{2}$ raised a number of important points on cSS definition and detection and opined that the pathogenesis of cSS in the context of CAA is still an unproven hypothesis. They suggested that an, as yet, unidentified bleeding source may account for cSS in the studied cohort instead of CAA. ${ }^{2}$ Some of these issues deserve further attention. First, ample evidence suggests that cSS detected in older patients (generally older than 50 years of age) in the context of small-vessel disease (including CAA) is quite distinct from the "classic" superficial siderosis of the central nervous system with regard to underlying pathology, pattern, and clinical presentation. ${ }^{3}$ The classic superficial siderosis of the central nervous system (to which Bai et al are referring), affects mainly the infratentorial regions (brain stem and posterior fossa) and spinal cord and typically presents with progressive sensorineural hearing loss, cerebellar ataxia, and corticospinal tract signs. ${ }^{3}$ By contrast, published CAA cases with cSS lack these typical clinical manifestations, and cSS is limited to the supratentorial compartment over the convexities of the cerebral hemispheres (almost always sparing the brain stem, cerebellum, and spinal cord). ${ }^{4,5}$ It is possible the use of the term "siderosis" for both entities is contributing to this confusion.

While I completely agree with Bai et $\mathrm{al}^{2}$ that cSS has a wide variety of potential causes, in older individuals, it is emerging as a key neuroimaging signature of CAA. In fact, revised diagnostic criteria for CAA include cSS as an additional hemorrhagic manifestation of the disease, equivalent to lobar cerebral microbleeds and intracerebral hemorrhage. ${ }^{4}$ The exact pathophysiologic

http://dx.doi.org/10.3174/ajnr.A4748 mechanisms of the association between cSS and CAA remain an active field of research. However, the role of CAA-related microangiopathic disease as an important cause of cSS in the elderly needs to be emphasized; the most biologically plausible hypotheses based on available evidence are extensively discussed in a recent expert review. ${ }^{5}$

Some technical issues on cSS detection and evaluation are also mentioned by Bai et $\mathrm{al}^{2}$ in their letter. Most important, differentiating cSS from cortical microbleeds is not difficult if operational criteria are applied because the 2 lesions have different size ranges

\section{Updated minimum criteria for identification of cortical superficial siderosis and acute convexity subarachnoid hemorrhage in the context of CAA and small-vessel disease ${ }^{5}$ \\ Criteria}

- Well-defined, homogeneous hypointense curvilinear signal intensity (black) on T2*-GRE or SWI MRI in the superficial layers of the cerebral cortex, within the subarachnoid space, or in both

- Blooming effect on T2*-GRE and SWI compared with T1- or T2-weighted sequences

- Differentiation from multiple very superficial cortical cerebral microbleeds (small, generally 2-5 mm, well-defined, homogeneous, and either round or oval lesions, at least half surrounded by brain parenchyma)

- If there is corresponding signal hyperintensity in the subarachnoid space on proton density-weighted or FLAIR sequences (or hyperdensity on CT, if available), the term "acute cSAH" should be used

- Axial T1-weighted or FLAIR images should be used for anatomic confirmation of the gyral location of the signal hypointensities identified on T2*-GRE or SWI sequences

- Absence of infratentorial (brain stem, cerebellum, spinal cord) siderosis

- Ensure exclusion of potential hemorrhagic and nonhemorrhagic cSS mimics (eg, vessel flow voids, thrombosed vessels, petechial hemorrhagic transformation of infarcts, calcium deposits)

- Consider all potential non-CAA secondary etiologies of cSS and acute cSAH

- cSS should be categorized as focal or disseminated (eg, in line with the modified Boston criteria)

- In each patient, the location (cerebral lobes and so forth) of cSS and the number of cerebral sulci affected can be recorded

- Other relevant vascular neuroimaging lesions both remote from and in close proximity $(\mathrm{eg}$, up to $1 \mathrm{~cm})$ to cSS should be evaluated, using established standards (eg, cerebral microbleeds, acute small DWI lesions, and so forth)

Note:-GRE indicates gradient recalled-echo; $\mathrm{CSAH}$, convexity $\mathrm{SAH}$ 
and shapes on $\mathrm{T} 2{ }^{*}$-weighted gradient-echo or susceptibilityweighted imaging sequences. cSS shows curvilinear signal intensity in the superficial layers of the cerebral cortex, within the subarachnoid space, or in both, whereas microbleeds are small (generally 2-5 mm), well-defined, homogeneous, and either round or oval lesions. ${ }^{6}$ In addition, at least half of the microbleed lesion should be surrounded by brain parenchyma-unlike cSS. The differentiation of microbleeds and cSS becomes more challenging when (rarely) multiple microbleeds are located very near the cortical surface, creating "microbleeds pearls." In this scenario, the systematic application of operational criteria is essential. Hence, Bai et al touch on a key methodologic aspect in smallvessel disease imaging marker research, namely the use of consistent imaging criteria and standards for evaluating and reporting cSS in relevant studies in the field. To facilitate this, we have recently suggested definitions and minimum imaging standards for cSS evaluation ${ }^{5}$ - a slightly modified version of these is provided in the Table. Further studies on the sensitivity and specificity of cSS as a diagnostic MR imaging marker of CAA are necessary.

\section{REFERENCES}

1. Inoue $Y$, Nakajima M, Uetani $\mathrm{H}$, et al. Diagnostic significance of cortical superficial siderosis for Alzheimer disease in patients with cognitive impairment. AJNR Am J Neuroradiol 2015 Oct 8. [Epub ahead of print] CrossRef Medline

2. Bai HX, Zhou H, Tan X, et al. Cerebral amyloid angiopathy as an etiology for cortical superficial siderosis: an unproven hypothesis. AJNR Am J Neuroradiol 2015 Dec 31. [Epub ahead of print] CrossRef Medline

3. Linn J, Brückmann H. Superficial siderosis in cerebral amyloid angiopathy. AJNR Am J Neuroradiol 2010;31:E29 CrossRef Medline

4. Linn J, Halpin A, Demaerel P, et al. Prevalence of superficial siderosis in patients with cerebral amyloid angiopathy. Neurology 2010;74: 1346-50 CrossRef Medline

5. Charidimou A, Linn J, Vernooij MW, et al. Cortical superficial siderosis: detection and clinical significance in cerebral amyloid angiopathy and related conditions. Brain 2015;138:2126-39 CrossRef Medline

6. Greenberg SM, Vernooij MW, Cordonnier C, et al; Microbleed Study Group. Cerebral microbleeds: a guide to detection and interpretation. Lancet Neurol 2009;8:165-74 CrossRef Medline

(1D) A. Charidimou J. Philip Kistler Stroke Research Center Department of Neurology Massachusetts General Hospital Stroke Research Center Harvard Medical School Boston, Massachusetts 